Canadian Journal of Higher Education Revue canadienne d'enseignement supérieur

Volume 42, No. 3, 2012, pages 45-64

\title{
Typologie des conceptions des universités en vue d'en évaluer la performance : rendre compte de la diversité pour en saisir la complexité
}

Catherine Larouche

Université du Québec à Chicoutimi

Denis Savard, Lucie Héon et Jean-Joseph Moisset

Université Laval

\section{RÉSUMÉ}

Le système universitaire est complexe et subit de nombreuses pressions pour qu'on évalue sa performance. Comment définir la performance des universités? Il existe une diversité d'opinion quant au choix des dimensions, des critères et des indicateurs à privilégier. Cet article a pour but de présenter une typologie des conceptions des universités en vue d'évaluer leur performance. À partir d'une recension des écrits, un prototype de la typologie a été élaboré, lequel comporte sept conceptions des universités. De manière à valider cette typologie, un processus d'anasynthèse (Legendre, 2005; Sauvé, 1992; Silvern, 1972) a été utilisé. Onze experts ont été rencontrés dans le cadre d'entretiens semi-structurés. Ces experts ont porté un jugement sur la typologie proposée en fonction de six critères de validation (clarté, consistance logique, exhaustivité, économie, utilité et acceptabilité par les usagers). L'article sous référence présente les résultats de cette recherche, de même que les sept types de typologie optimale (service public, marché, académique, apprenante, politique, entrepreneuriale et milieu de vie). 


\section{ABSTRACT}

The university system is complex and is constantly pressured to evaluate its performance. How should university performance be defined? There is no agreement on the dimensions, criteria and indicators to choose. This article presents a typology of the conceptions of universities so as to evaluate their performance. Based on an extensive literature review, a typology prototype consisting of seven conceptions of universities was developed. A method of anasynthesis (Silvern, 1972; Sauvé, 1992; and Legendre, 2005) was used to verify the typology. Semi-structured interviews were held with eleven experts. These experts assessed the proposed typology in relation to six validation criteria (clarity, logic consistency, comprehensiveness, economy, usefulness and acceptability by users). This article presents the results of this research as well as the seven optimal typology categories (public service, market, academic, learner, political, entrepreneurial and living environment).

\section{PROBLÉMATIQUE}

Plus que jamais, les universités subissent des pressions pour qu'elles évaluent leur performance ${ }^{1}$ : des pressions gouvernementales (gestion par résultat, reddition de compte), des pressions internationales (mobilité, compétition, reconnaissance, accessibilité), mais aussi des pressions en provenance des communautés internes et externes des établissements (qualité, amélioration, palmarès, transparence) (CSE, 1999).

Dans leurs pratiques de suivi et d'évaluation, la plupart des universités n'utilisent généralement qu'un nombre restreint d'indicateurs qui ne dévoilent qu'une vision partielle de leur performance (Tavenas, 2004). Les opérations d'évaluation répondent à des initiatives principalement externes qui privilégient l'uniformité en vue de la comparaison et qui favorisent la reddition de compte par rapport au processus d'amélioration continue (Savard, Larouche, \& Héon, 2004; Tavenas, 2004). Les indicateurs utilisés découlent pour la plupart d'une conception économique ou sont inspirés des méthodes de l'entreprise privée.

L'objectif principal de cet article est de présenter une typologie des conceptions ${ }^{2}$ des universités en vue d'évaluer leur performance, typologie issue d'une recherche doctorale. Cette typologie permet une meilleure lecture de la complexité du système universitaire et de son fonctionnement, en proposant des critères d'évaluation en fonction des différentes façons de définir la performance des universités.

La mission fondamentale des universités repose sur la production, la création, la conservation, la transmission et la diffusion des savoirs. Leurs activités d'enseignement, de recherche et de services aux collectivités constituent le socle commun autour duquel les universités ont édifié leur spécificité.

Les universités constituent des organisations complexes. Elles sont composées d'acteurs multiples, à différents niveaux, avec des agendas, des valeurs et des cadres de référence divers provenant tant de l'intérieur que de l'extérieur de l'institution. Ces acteurs doivent coordonner leurs actions, échanger de l'information et interagir dans des dynamiques qui n'excluent pas la concurrence (Héon, 1991), l'antagonisme et les contradictions. Par conséquent, les résultats de leurs actions ne sont pas " linéaires », ce qui leur a valu 
d'être qualifiées de bureaucratie professionnelle (Mintzberg, 1990), d'anarchie organisée (Cohen, March \& Olsen, 1972) ou de couplage souple ${ }^{3}$ (Weick, 1976).

À la mission fondamentale des universités se greffent, pour chacun des établissements, des objectifs spécifiques. Selon les époques et les circonstances, l'université remplit sa mission différemment, dans un contexte et des conditions qui évoluent et se transforment (Rocher, 1990).

La mission fondamentale est traversée et colorée par différentes conceptions des universités. Ces conceptions reposent sur des valeurs et des principes qui amènent à définir différemment l'enseignement, la recherche, la gouvernance et la performance, ainsi qu'à privilégier des moyens d'évaluation ${ }^{4}$ et des indicateurs distincts. Les établissements universitaires sont appelés à poursuivre des objectifs en apparence antagoniques comme, par exemple, assurer l'accessibilité du plus grand nombre tout en sélectionnant les meilleurs pour former une élite, s'assurer de la transmission des savoirs tout en poussant plus loin la recherche de nouvelles connaissances, maintenir la recherche fondamentale tout en soutenant la recherche commanditée.

Or, comment définir la performance des universités en l'absence de consensus sur la définition de leur performance? Quels sont les critères de performance à privilégier? Les étudiants peuvent préférer celui de l'accès à l'éducation; les conseils d'administration des universités, celui de l'équilibre budgétaire; les syndicats cherchent à obtenir de meilleures conditions de travail; le ministère de l'Éducation demande à ce que l'on augmente les taux de diplomation; les associations professionnelles cherchent à obtenir de meilleurs résultats aux examens de promotion. Quel modèle d'évaluation peut tenir compte des différentes conceptions ambiantes?

Notre démarche s'est appuyée sur l'élaboration d'une typologie des conceptions des universités. Les types de conception des universités ont été identifiés selon une approche interprétative (Bonniol \& Vial, 1997; Mintzberg, Ahlstrand, \& Lampel, 1999; Morgan, 1999). Nous avons postulé qu'à l'intérieur des établissements universitaires coexistent une pluralité de conceptions qui varient selon des rythmes d'alternance et de prédominance, dans le temps, dans l'espace et selon les individus. L'établissement universitaire s'avère ainsi un point de rencontre spécifique d'un certain nombre de conceptions diversifiées qui y sont privilégiées.

S’il existe effectivement différentes conceptions des universités, comment alors les identifier et parvenir à des consensus quant aux critères et aux indicateurs propres à évaluer leur performance? La littérature du domaine des organisations ouvre la voie à l'élaboration de modèles typologiques ${ }^{5}$.

Après l'analyse critique de quelques typologies de mesure de la performance organisationnelle, principalement celles utilisées pour les entreprises (Morin, Savoie, \& Baudin, 1994; Quinn \& Rohrbaugh, 1983), force est de constater que ces modèles s'appliquent difficilement aux universités puisque celles-ci ne peuvent être considérées comme des entreprises au sens où on l'entend dans le domaine privé (Freitag, 2002). La prise en compte, à la fois de la complexité ${ }^{6}$ et de la diversité des universités appelle donc un modèle d'évaluation plus élaboré, doté d'un ensemble d'indicateurs plus riche et plus pertinent. Le défi consiste à trouver des modèles qui permettent de rendre compte de cette diversité et de cette complexité. 
Pour définir la performance des universités, il fallait d'abord plonger au cœur de leur réalité et dégager les conceptions qui y sont présentes pour décrire ces établissements. C'est dans cette perspective qu'ont été établis les objectifs de la recherche, soit :

- élaborer une typologie des conceptions des universités en vue d'en évaluer la performance selon une approche différenciée;

- valider la typologie élaborée auprès d'experts de la gouvernance des universités et des administrations publiques.

\section{MÉTHODOLOGIE}

Cette recherche de type méthodologique (Kerlinger, 1973) est fondée sur un processus d'anasynthèse (Sauvé, 1992; Legendre, 2005; Silvern, 1972). Selon Fortin (1996, p. 203), " la recherche méthodologique se définit comme une stratégie en plusieurs étapes portant sur la mise au point ou la validation d'un instrument de mesure nouvellement créé ou traduit dans une autre langue ». L'anasynthèse est un néologisme formé des mots analyse et synthèse désignant le processus général d'élaboration des modèles. Il s'agit d'un " processus cyclique et cybernétique qui implique l'analyse d'une situation de départ, l'élaboration d'une synthèse à partir de cette analyse, la formulation d'un prototype de modèle, la simulation ou validation de ce prototype en vue finalement de la formulation d'un modèle optimal » (Legendre, 2005; Sauvé, 1992). Cette méthode est schématisée à la figure 1.

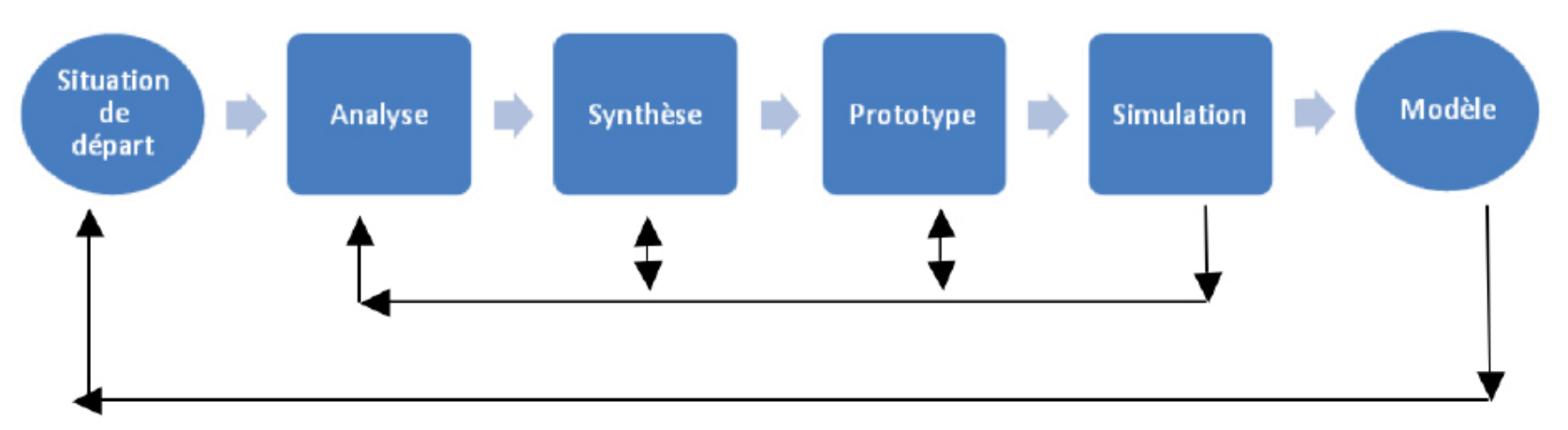

Figure 1. Le processus d'anasynthèse adaptation de Silvern (1972), par Legendre (2005).

Nous appuyant sur une recension exhaustive des écrits sur la question, nous avons mis en application un processus d'anasynthèse consistant, d'une part, à l'analyse des modèles de mesure de performance des universités et, d'autre part, à la synthèse des conceptions qui leur sont sous-jacentes en vue de faire ressortir une typologie préliminaire. Cette typologie préliminaire des conceptions des universités a été soumise au jugement de trois experts ${ }^{7}$ dans le cadre d'une étude pilote, en décembre 2007. Les rétroactions obtenues à ce stade ont permis d'élaborer un prototype de la typologie. L'étape de " simulation » a servi à valider le prototype et à y proposer les modifications jugées nécessaires. Onze experts ont ainsi été rencontrés, de mai à novembre 2008, dans le cadre d'entretiens semistructurés, jusqu'à saturation des données. Les entretiens ont été analysés en fonction des six critères de validité d'une typologie selon Sauvé (1992) : la clarté, la consistance logique, 
l'exhaustivité, l'économie, l'utilité et l'acceptabilité par les usagers. Les propositions issues des entretiens ont été soumises à un comité de validation ${ }^{8}$, de mars à octobre 2009 , qui a arbitré les propositions, accepté les modifications finales et proposé une typologie optimale (le modèle).

À la suite des modifications apportées, les sept conceptions proposées ont été jugées assez claires par les experts (moyenne 2,9/4 sur une échelle de clarté) et claires par le comité de validation (moyenne 3,53/4). La typologie a été jugée exhaustive par une majorité d'experts. Deux experts ont proposé l'ajout d'une huitième conception : une conception gestionnaire qui n'a pas été retenue par le comité de validation, mais qui a tout de même été jugée assez pertinente pour faire l'objet d'une éventuelle validation subséquente. Bien que certains experts aient proposé la fusion ou le retrait de certaines conceptions, le comité de validation a maintenu la structure de la typologie préliminaire en y intégrant plusieurs des modifications suggérées lors de l'enquête. Les experts ont proposé plusieurs réalités signifiantes pour illustrer chacune des conceptions. Tous les experts ont jugé la typologie utile dans plusieurs contextes et susceptible d'être acceptée par les usagers, en particulier par les responsables de la gouvernance d'établissements universitaires et ceux appelés à évaluer la performance de ces établissements.

\section{LA TYPOLOGIE}

La recherchea conduità unetypologieoptimale(lemodèle)comportantseptconceptions (service public, marché, académique, apprenante, politique, entrepreneuriale et milieu de vie). Ces conceptions sont définies en fonction de sept dimensions (valeurs et principes, système de gouvernance, stratégies, recherche, enseignement, évaluation et critères de performance).

Chaque conception repose sur des valeurs et des principes, lesquels permettent de la définir et de désigner les axes fondamentaux autour desquels s'établit son échelle de priorités. Le choix de relier gouvernance, stratégies et évaluation de la performance permet de rattacher les critères de performance à des intentions de gouvernance. La mission fondamentale des universités, leur socle commun, est traversée par les différentes conceptions tout en laissant place à une interprétation nuancée, qui suit les conceptions dominantes et partagées.

Il est à noter que les conceptions proposées se distinguent des types "idéaux », au sens de types " purs », dont les caractéristiques seraient mutuellement exclusives. Il convient également de préciser qu'il ne s'agit pas de types d'université, mais bien de types de conception des universités; une même université peut ainsi s'inscrire dans plus d'un type de conception.

On retrouve, au tableau 1, la description de la typologie optimale et les conceptions des universités qui la composent. 
Typologie des conceptions des universités / C. Larouche, D. Savard, L. Héon \& J-J Moisset 50

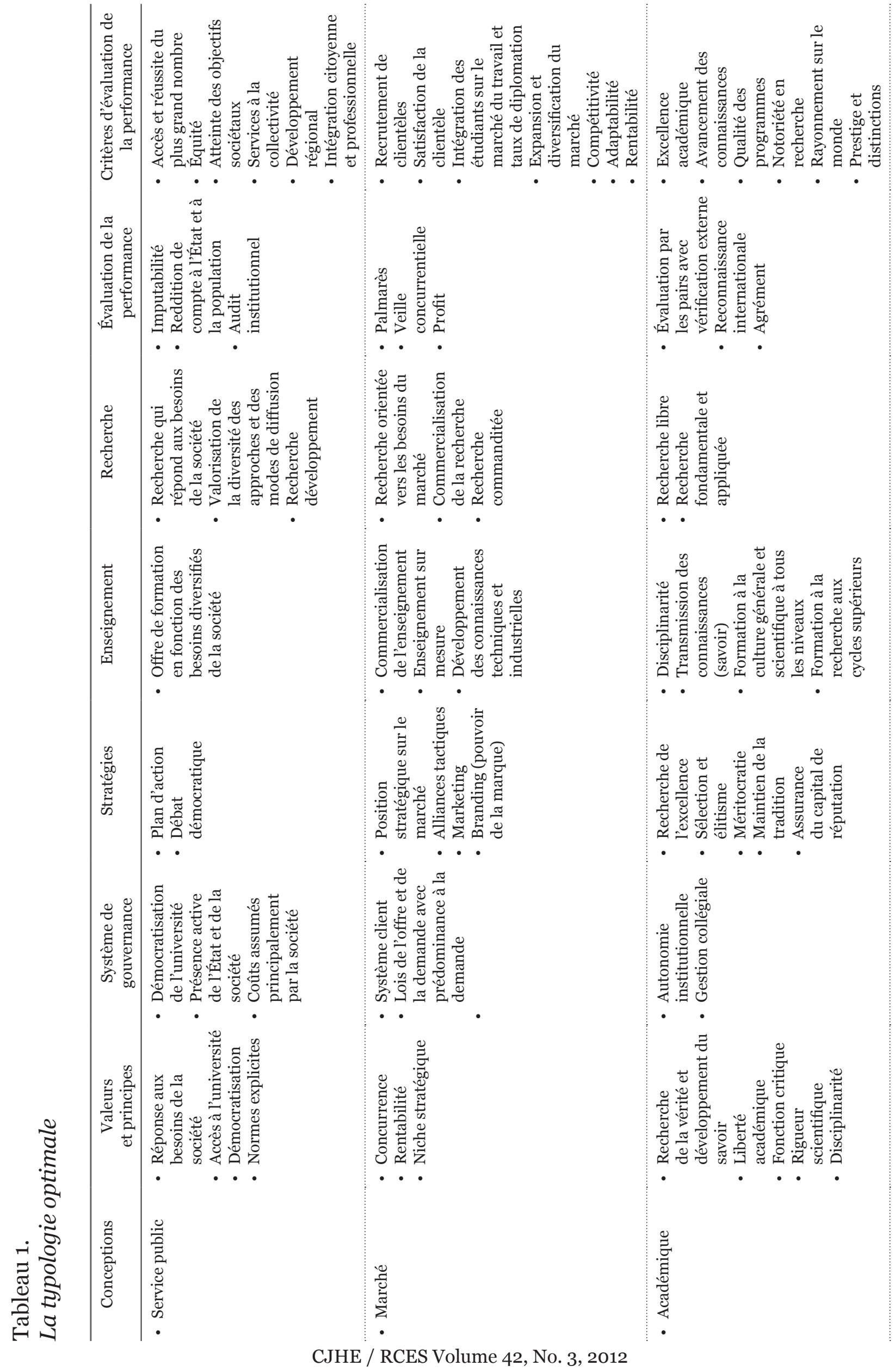


Typologie des conceptions des universités / C. Larouche, D. Savard, L. Héon \& J-J Moisset 51

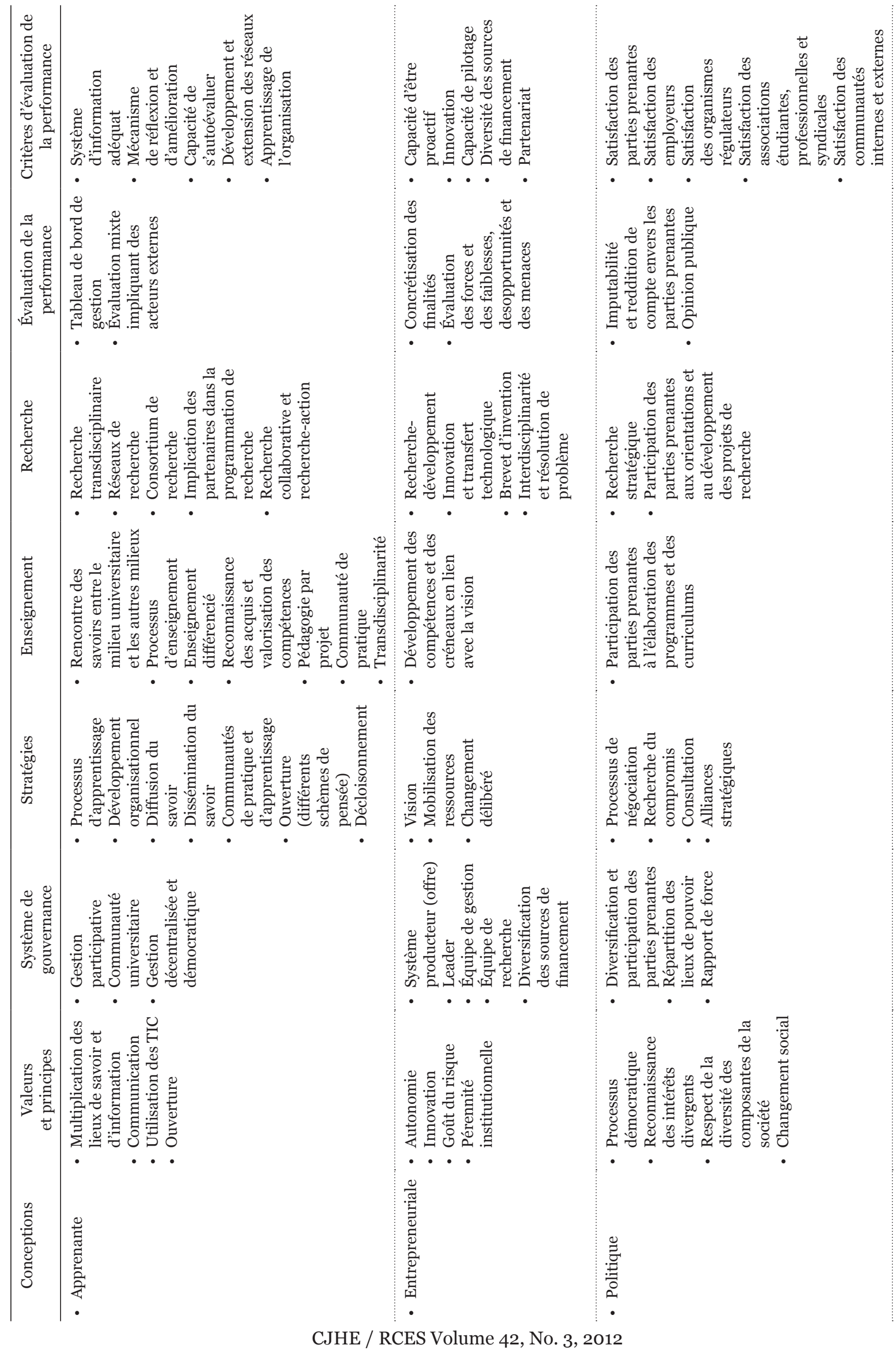


Typologie des conceptions des universités / C. Larouche, D. Savard, L. Héon \& J-J Moisset 52

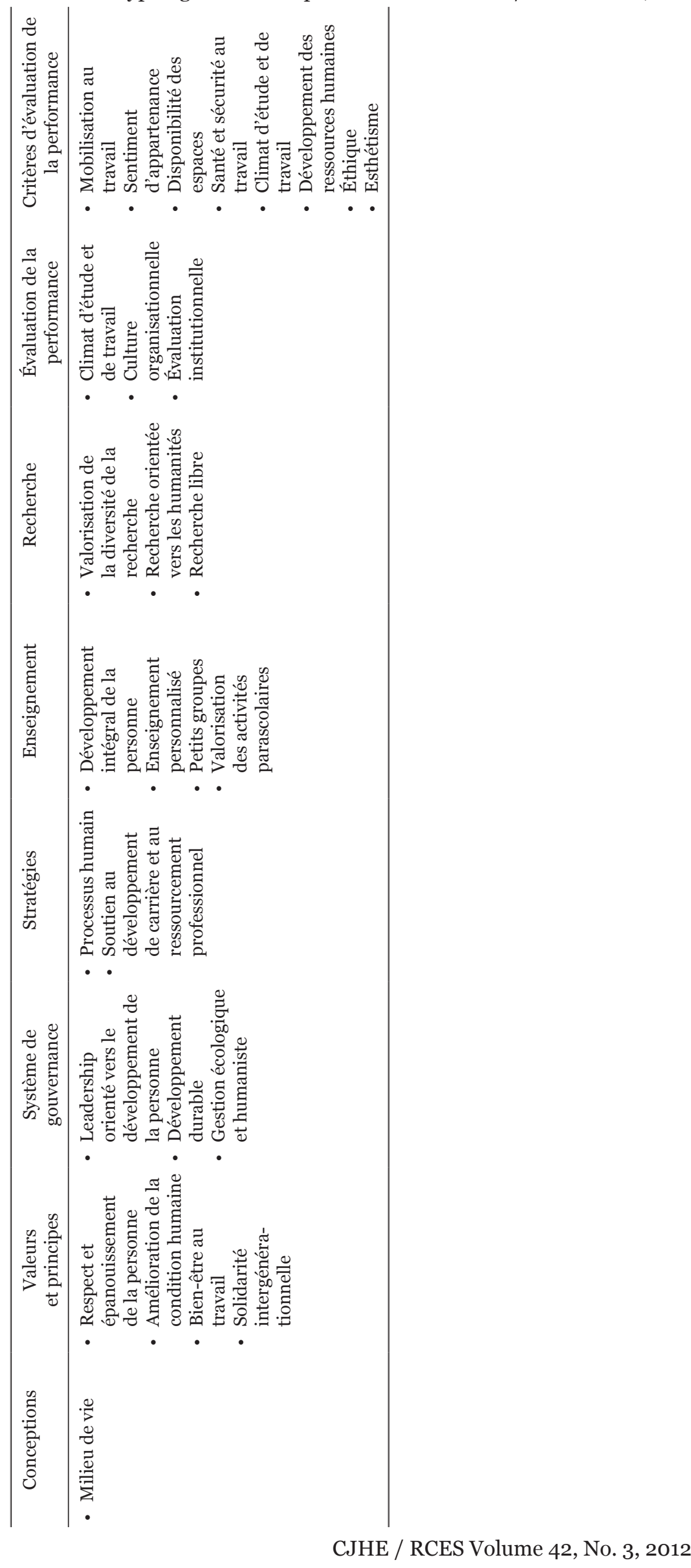


Dans les sections qui suivent, les conceptions retenues dans la typologie sont brièvement décrites en fonction de leurs principales caractéristiques. Sont ainsi présentées, dans l'ordre, les conceptions service public, marché, académique, entrepreneuriale, politique, apprenante et milieu de vie.

\section{La conception service public}

La Politique québécoise des universités (MEQ, 2000, p. 17) définit l'université comme un service public. Cette politique stipule que « l'éducation, l'enseignement supérieur et spécialement la formation universitaire constituent un bien personnel et social. »

En 1997, la Fédération québécoise des professeures et professeurs d'université (FQPPU) proposait la définition suivante de l'université comme service public :

Le point d'ancrage et le socle de cette définition, c'est l'université au service de toutes et de tous, l'Université du bien commun et du patrimoine collectif, l'université de la réussite et de la qualification, ce n'est pas l'université-client, c'est l'universitécitoyen. Cette définition qu'il faut élaborer et proposer, c'est l'université comme service public. [...] C'est une université ouverte sur la société capable de répondre à ses attentes et contribuant au progrès de la collectivité dans son ensemble. (FQPPU, 1997, p. 1 et 4)

Pour les tenants de cette conception, les universités appartiennent à la sphère du Bien commun (Lucier, 2006). Elles contribuent ainsi au progrès de la collectivité dans son ensemble. Au Québec, il faut éviter de confondre service public et service étatique. Sous la conception de service public proposée, l'université n'est pas complètement décentralisée comme le préconise le modèle américain, ni centralisée comme dans le modèle français. Les coûts de l'institution québécoise sont assumés principalement par la société et elle est régie par des normes d'équité explicites9.

Dans la conception de service public, on identifie et on priorise les besoins d'abord, puis on planifie l'action en conséquence pour y répondre de façon adéquate et équitable. Les tenants de cette conception prônent le débat démocratique et la participation de la société dans la définition des objectifs. Pour assurer l'accès au plus grand nombre de citoyens, l'université offre une formation qui est à la fois diversifiée à tous les cycles universitaires et adaptée aux besoins de la société. Elle privilégie une recherche contextualisée qui vise notamment à répondre aux besoins sociaux. La rencontre de la théorie et de l'action correspond à une nécessité sociale, tant pour l'étudiant que pour le chercheur qui fait de la recherche un foyer de progrès social.

Dans une perspective de service public, on évalue la performance pour rendre des comptes à la population et au gouvernement parce que les acteurs du système universitaire sont imputables. Sur le plan de la gestion, l'imputabilité s'actualise dans des exigences de transparence auprès des citoyens. Les principaux critères caractérisant la conception de service public sont : l'atteinte des objectifs sociétaux, l'équité, les services à la collectivité, le développement régional, l'accès et la réussite du plus grand nombre, et l'intégration citoyenne et professionnelle des étudiants à la société. 


\section{La conception marché}

Pour les tenants de la conception de marché, l'université est vue comme ayant une valeur marchande (Porter, 1993) soumise aux lois d'un marché autorégulateur selon une logique identifiable aux théories d'Adam Smith. Avec l'avènement de la pensée néolibérale, c'est l'intérêt ou le désir d'acquisition qui agit comme force organisatrice de la société. La logique de marché se présente dans les établissements universitaires par l'idée de la capacité à développer l'innovation afin de répondre à une demande déterminée dans un contexte de concurrence régionale, nationale et internationale. L’idée de marché s'articule principalement autour des questions de positionnement concurrentiel et de satisfaction de la clientèle, tant sur le plan de l'enseignement que sur celui de la recherche et des services à la collectivité.

Dans une conception de marché, l'université s'adapte aux besoins de sa clientèle. Il s'agit donc d'un contrôle externe; une bonne part du pouvoir se situe à l'extérieur des murs de l'établissement. Cette dévolution du pouvoir à l'externe s'exprime par l'attribution de sièges réservés à des entreprises privées au sein des instances décisionnelles ou par des mécanismes de gouvernance qui requièrent la prise en compte explicite des besoins de la clientèle.

Dans une telle perspective, l'université utilise des stratégies issues du marketing pour promouvoir sa marque de commerce auprès de sa clientèle cible. L'université tente ainsi d'améliorer sa position sur le marché en créant des niches stratégiques. Les tenants de la conception de marché privilégient un enseignement sur mesure et une recherche commanditée qui répond à des besoins spécifiques en provenance des entreprises (Crespo \& Dridi, 2007). Évaluer la performance en fonction de cette conception consiste, entre autres, à effectuer une veille concurrentielle et à se prêter au jeu des palmarès d'établissements. Les critères d'évaluation de performance privilégiés ici sont : le recrutement et la satisfaction des étudiants, le taux de diplomation, l'intégration des diplômés sur le marché du travail, la compétitivité, l'adaptabilité et la rentabilité.

\section{La conception académique}

Alors que ceux qui adhèrent aux conceptions de service public et de marché cherchent à adapter leur fonctionnement à l'évolution de leur environnement - tout en répondant, respectivement, aux besoins de la société et du marché -, les tenants de la conception académique cherchent plutôt à atteindre un idéal. Ils tentent en effet d'orienter le mouvement général de la société. Selon Freitag (2002), l'université porte et exprime certaines finalités idéales qui transcendent la temporalité et l'espace. Ainsi, dans la conception académique, l'université n'est pas au service de l'économie, elle concrétise plutôt une idée civilisatrice qui vise la formation des citoyens à un idéal d'humanité, de culture et de vie commune (Freitag, 2002). Cette idée d'humanité, qui repose sur l'émancipation de l'individu et de la société dans leur globalité, prend ses distances visà-vis des dogmatismes religieux, des pouvoirs politiques et des besoins du marché. La conception académique sous-tend ainsi une proposition normative différente quant au développement de la société et des personnes qui la composent. On y recherche la vérité, on valorise le développement du savoir et la rigueur scientifique. Au sein de cette conception, la liberté académique se révèle être une valeur fondamentale. 
La fonction critique de l'université est garantie par la liberté académique et par l'autonomie institutionnelle. Cette liberté et cette autonomie se vivent dans un contexte de gestion collégiale, c'est-à-dire par la participation de la communauté universitaire à tous les niveaux et à toutes les instances de gestion. La gestion collégiale s'oppose ainsi fondamentalement au modèle hiérarchique de gestion. Dans la conception académique, l'université recherche l'excellence, elle favorise le maintien de normes de qualité élevées, préconise la méritocratie et cultive son prestige. Les tenants de cette conception privilégient une formation à la culture générale et scientifique, à tous les niveaux d'enseignement. La recherche libre et fondamentale se trouve essentiellement gouvernée par la communauté académique et répond à des intérêts axés notamment sur le développement de nouvelles connaissances disciplinaires.

Pour les tenants de la conception académique, l'évaluation de la performance doit respecter le principe d'autonomie universitaire et privilégier des critères tels que l'excellence académique, la qualité des programmes, la notoriété en recherche, la diversité de la recherche, le rayonnement sur le monde, le prestige et les distinctions.

\section{La conception entrepreneuriale}

Pour les tenants de la conception entrepreneuriale, l'université s'inscrit dans un mode de gestion qui vise à exploiter les ressources et les possibilités de développement au-delà des moyens disponibles (Clark, 1998). Dans cette conception, l'établissement se charge lui-même de se gouverner, de se réglementer. Il s'adapte à un univers complexe et incertain, mais le fait à ses propres conditions.

La conception entrepreneuriale se distingue de la conception de marché dans la mesure où elle repose sur la logique de l'offre, qui est marquée par le goût du risque et la créativité et se concrétise par des productions novatrices, alors que la logique de la conception de marché se préoccupe de la demande et se traduit par des productions adaptées.

Pour Clark (2001), l'autonomie institutionnelle implique que l'université ne penche ni pour l'État, ni pour le marché. Les tenants de la conception entrepreneuriale veulent donc mettre en évidence la coordination professionnelle des équipes d'enseignants et d'administrateurs qui fonctionnent selon leurs propres normes de responsabilité et de transparence. Ils se soucient du développement d'une identité distinctive et prônent une culture intégrée. Dans cette perspective, on valorise la mobilisation des ressources autour d'une vision porteuse de changement. On vise en outre le développement des compétences et des créneaux de recherche en lien avec cette vision, tout en privilégiant la recherchedéveloppement, l'innovation et le transfert technologique.

Dans la conception entrepreneuriale, on s'éloigne de la conformité aux plans communs et aux visions gouvernementales auxquels on préfère une évaluation adaptée à la spécificité des établissements, en fonction deleurs finalités propres. Les critères d'évaluation privilégiés par les tenants de cette conception sont : la capacité d'être proactif, l'innovation, la capacité de pilotage, la diversité des sources de financement et le nombre de partenariats.

\section{La conception politique}

Les tenants de la conception politique recherchent la légitimité auprès des parties prenantes (Morin, Savoie, \& Beaudin, 1994; Quinn \& Rohrbaugh, 1983). Il ne s'agit pas ici de s'adapter aux besoins du marché comme le voudraient ceux qui adhèrent à 
la conception de marché, ni de s'adapter aux besoins de la société comme le prônent les tenants de la conception de service public, mais d'obtenir le soutien et la confiance des acteurs de son environnement. De plus en plus, les organisations et les universités doivent se préoccuper de leur légitimité sociale au-delà des considérations du marché. Les tenants de la conception politique veulent mettre l'accent sur le changement social en luttant contre les forces dominantes et en cherchant l'équité entre les différentes composantes de la société. Au sein des universités, les enjeux relatifs à l'enseignement supérieur et à la recherche scientifique sont très importants. Ils sont nécessairement soumis aux forces politiques internes et externes des institutions. De fait, il est difficile sinon impossible d'empêcher que des groupes d'intérêts variés cherchent à exercer et exercent effectivement une influence sur la détermination des choix dans ces domaines. Les tenants de cette conception favorisent la participation des parties prenantes dans la définition des programmes et des curricula, de même que dans l'élaboration et la gestion des orientations de recherche. Il revient néanmoins aux responsables de la gouverne des universités de préserver leur mission fondamentale en trouvant un certain équilibre entre ces groupes d'intérêts.

Cette perspective met en évidence les conflits d'intérêts et la recherche de la satisfaction du plus grand nombre de constituantes. La conception politique favorise comme moyens d'évaluation : la satisfaction, l'imputabilité et la reddition de compte envers les parties prenantes. Les tenants de cette conception privilégient principalement le critère de satisfaction des parties prenantes.

\section{La conception apprenante}

Depuis quelques années, on voit apparaitre dans la littérature sur l'administration universitaire le concept d'organisation apprenante (Duke, 1992; James, 2000; Mead, 1995; Ramsden \& Martin, 1996). Pour Dill (2003) et Garvin (1993), l'organisation apprenante est une organisation habilitée à créer, à acquérir et à transférer des connaissances, ainsi qu'à modifier sa conduite pour refléter de nouvelles connaissances et perspectives. Nous ajouterons que cette conception répond bien au phénomène connu depuis longtemps de l'obsolescence, vieillissement prématuré des connaissances, mais devenu encore plus important avec le développement exponentiel des inventions et des innovations technologiques.

Dans le cas de cette conception, on parle du passage de la gestion hiérarchique de l'information à une gestion horizontale et diffuse de celle-ci. Les organisations ne sont apprenantes que dans la mesure où les membres des groupes qui les composent témoignent individuellement et collectivement de leur désir d'apprendre et acceptent de se considérer en situation de formation permanente.

Les tenants de la conception apprenante prônent la démocratisation, la participation, la gestion collégiale et la contribution des différents groupes à la création et à la vie du milieu universitaire. Cette perspective privilégie la mise en place de structures de réflexion collective et de rétroaction (les communautés de pratique, d'apprentissage et de recherche). Mettant généralement à profit les avancées technologiques, une université où la conception apprenante prédomine favorise la diffusion et la dissémination du savoir, le décloisonnement des structures ainsi que l'utilisation de différents schèmes de pensée pour la résolution de problème. 
L’organisation apprenante convie au travail en équipe et en réseaux, à la communication des savoirs tacites et formels de même qu'elle amène les acteurs à expliciter leurs valeurs (Askling \& Kristensen, 2000; Bouvier, 2007; Harvey \& Knight, 1996; Srikanta \& Dalrymphe, 2002). La coopération invite aux débats d'idées pour passer à la phase suivante, celle de la construction et de la mobilisation des compétences autour d'un projet commun, toujours en mouvement.

Si les tenants de cette conception s'opposent aux théories de la gestion scientifique, ils ne rejettent pas moins une transmission linéaire des savoirs, considérant leur vision de l'enseignement. Ainsi, la formation de l'esprit ne passe pas seulement par la familiarisation avec les œuvres classiques ou les connaissances scientifiques, mais aussi par une capacité d'analyse, de mise en relation, de questionnement et de transposition (Perrenoud, 1995). Sur le plan de l'enseignement, les tenants de la conception apprenante expriment une préoccupation plus grande pour le transfert des connaissances. De même, ils considèrent que la pédagogie doit s'adapter constamment à l'évolution des processus d'apprentissage ainsi qu'aux modes de penser, d'agir et d'apprendre des nouvelles générations. Les compétences renvoient à des savoir-faire de haut niveau qui exigent l'intégration de multiples ressources cognitives dans le traitement de situations complexes. Le type de recherche scientifique préconisé dans la conception apprenante est lié aux besoins sociaux et sollicite l'implication de partenaires dans sa programmation. Cette perspective favorise la recherche collaborative et la recherche-action, la recherche transdisciplinaire et la recherche en réseau, et elle valorise les consortiums de recherche.

Ainsi, les critères privilégiés par les tenants de cette conception sont la mise en place et l'exploitation de systèmes d'information efficaces, de mécanismes appropriés de réflexion, la capacité de s'autoévaluer, le développement des réseaux, l'apprentissage au sein de l'organisation.

\section{La conception milieu de vie}

La conception milieu de vie tire ses origines du courant humaniste qui prône que la pleine réalisation des capacités de l'homme transite par la connaissance de soi, la liberté d'agir sur sa propre destinée, la légitimité de la recherche du bonheur, la tolérance des idées diverses et un idéal commun de justice. Ici, le concept de culture organisationnelle se révèle important dans la mesure où celle-ci traduit une compréhension partagée du fonctionnement et des activités de l'organisation. Cette interprétation s'appuie sur un partage des traditions, des valeurs et des habitudes propres à la vie de l'organisation (Mintzberg, 1990). Dans les cultures organisationnelles qui supposent un fort sentiment d'appartenance, l'accent est mis sur la personne humaine et sur son apport à l'organisation, c'est-à-dire sur l'adhésion des membres aux finalités de l'organisation et aux comportements attendus d'eux. Dans cette perspective, la lecture cognitive de ce qu'il faut faire est partagée par une grande majorité sinon par tous, cette lecture étant évidemment susceptible d'évolution.

Pour les tenants de la conception milieu de vie, il importe d'investir dans les ressources humaines, d'adopter des politiques de gestion qui tiennent compte du type de travail et du type de carrière du personnel de haut niveau que l'on veut attirer et retenir, mais aussi de s'occuper des autres catégories d'employés : les professionnels techniques d'administration et de soutien, les étudiants, les représentants des partenaires externes. L'enseignement 
et la recherche privilégient le développement intégral de la personne, un enseignement personnalisé, la formation de petits groupes-classes, une valorisation des activités parascolaires et une recherche faisant une large place aux sciences humaines et sociales.

Ceux qui adhèrent à la conception milieu de vie privilégient l'évaluation du climat d'étude et de travail, la culture organisationnelle et l'évaluation institutionnelle. Ils accordent de l’importance aux critères suivants : la mobilisation des membres, le sentiment d'appartenance, la disponibilité des espaces, la santé et la sécurité au travail, le climat d'étude et de travail, le développement des ressources humaines, l'éthique et l'esthétisme.

\section{RÉSULTATS ET DISCUSSION}

Dans notre étude, nous avons mis en relief l'importance des travaux de Clark (1998) pour qui le système État, le système marché et le système professionnel, forment un triangle de tensions qui symbolise la recherche d'un équilibre entre ces trois intervenants. Ces trois systèmes s'opposent sur un continuum où chaque pointe représente la position extrême. La typologie optimale des conceptions de l'université issue de notre recherche apporte à cet égard un éclairage nouveau. Elle permet d'enrichir le modèle à trois axes en offrant la possibilité de lire la complexité de la réalité universitaire via les prismes de sept conceptions des universités qui la composent. Il est possible de concevoir que l'État, les responsables des établissements et ceux qui y travaillent, de même que les leaders de la société, puissent être porteurs d'une ou de plusieurs conceptions. Ainsi, le triangle des tensions évolue et devient un ensemble de cercles superposés et entrelacés représentant les conceptions simultanément présentes et nécessairement sous-tendues par la mission fondamentale de l'université. S'éloignant d'une vision unique et linéaire des universités, cette représentation telle qu'illustrée à la figure 2 montre bien toute la diversité et la complexité de la réalité dont il faut tenir compte lorsque vient le temps de choisir un cadre de référence en vue de l'évaluation de la performance des universités.

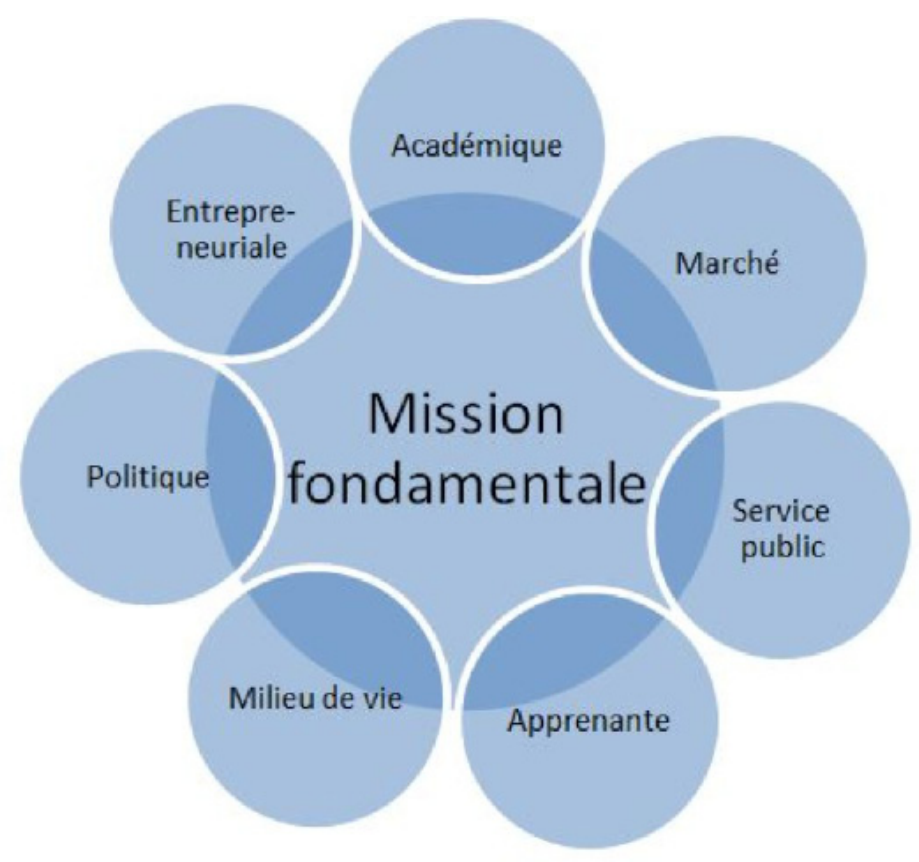

Figure 2. Le processus d'anasynthèse adaptation de Silvern (1972), par Legendre (2005). 
La figure 2 permet de constater que la mission fondamentale de l'Université est traversée par les sept conceptions. Le recours à des cercles permet d'illustrer que les types de conception possèdent leur propre identité, qu'elles sont distinctes les unes par rapport aux autres, sans être complètement mutuellement exclusives. Les cercles représentent aussi le mouvement d'évolution qui les anime dans le temps et dans l'espace.

La typologie optimale va aussi dans le sens d'autres modèles comme la typologie des sept mondes de la justification de Boltanski et Thévenot (1991) dont nous partageons les prémisses, à savoir le dépassement d'une opposition réductrice entre la sociologie holiste et l'économie individualiste. La réalité, plus complexe, peut se comprendre à travers le jeu des conceptions de notre typologie ou sous un certain nombre de formes de généralités, comme le proposent Boltanski et Thévenot.

La typologie permet de décrire les conceptions sans toutefois les hiérarchiser. Sur le plan de l'utilité des résultats, cette recherche est susceptible d'intéresser les chercheurs en gestion, les conseils d'administration des universités, les gouvernements, les gestionnaires universitaires, les professeurs, les étudiants et la population en général. Elle peut être considérée comme un outil de réflexion qui génère une meilleure lecture du réel des établissements universitaires. Elle peut être utilisée dans le cadre de processus de planification stratégique ou dans une optique d'évaluation institutionnelle. La typologie ouvre la voie à l'élaboration et à la mise en place de mécanismes inédits de pilotage et de reddition de compte mieux adaptés à la complexité et aux spécificités des universités. Elle peut servir de référentiel pour l'analyse critique des systèmes d'éducation et des modèles de régulation. Il sera également possible de s'en servir dans l'évaluation des politiques publiques pour apprécier les impacts des décisions prises en vue de l'atteinte de la mission, des buts et des objectifs institutionnels.

Quant aux pistes de recherche, il serait intéressant de mener cette même étude dans des contextes nationaux différents, notamment à l'aide d'études de cas. Des recherches ultérieures pourraient aussi servir à approfondir la distinction entre la conception marché et la conception entrepreneuriale, à mieux définir les conceptions apprenante et milieu de vie, de même qu'à valider la pertinence d'ajouter une huitième conception à la typologie, soit une conception gestionnaire.

\section{CONCLUSION}

En entreprenant cette recherche, notre intention était d'élaborer un modèle d'évaluation de la performance qui respecte la diversité et la complexité des établissements universitaires.

Bien que ces établissements partagent un socle commun qui détermine clairement qu'ils appartiennent à ce que l'on définit de manière générale comme étant l'« université ", ils peuvent être évalués, et leur performance mesurée, en fonction des conceptions dominantes partagées par ceux qui en sont responsables. À cet égard, nos résultats confirment que les sept conceptions des universités (service public, marché, académique, apprenante, politique, entrepreneuriale et milieu de vie) sont suffisantes pour rendre compte de la diversité des façons particulières d'évaluer la performance. N'offrant pas de lecture unifiée, ni une gamme fixe de critères, cette typologie optimale de sept conceptions est offerte à la communauté scientifique et aux praticiens comme référence en vue de soutenir une meilleure compréhension de la complexité et de la diversité universitaires. 
Il existe plusieurs façons de voir et de définir la performance, l'évaluation et la gouvernance des universités. En ce sens, les sept conceptions proposées sont susceptibles d'éclairer le choix des critères à privilégier dans le cadre d'évaluations. Les universités sont des systèmes complexes; sans prétendre répondre complètement au problème de la complexité, et encore moins de manière définitive, nous pensons que cette typologie permettra de mieux en lire la réalité. Car, au-delà de la gouvernance des universités, elle nous invite à tenir compte de la diversité et d'accepter la différence. Elle permet de mettre en relief les contradictions du réel, condition favorable pour se remettre en question. Enfin, et surtout, elle appelle à repenser l'avenir pour une société démocratique dans le respect de la pluralité des idées, des conceptions et des opinions minoritaires. S’il n'existe pas de modèle unique pour mesurer la performance des établissements universitaires, les résultats de la recherche présentée ici peuvent néanmoins aider les administrateurs universitaires et les gouvernements à créer des modèles de mesure de la performance mieux adaptés à la mission fondamentale des universités, à leur spécificité, et qui tiennent compte de la richesse et de la diversité de leurs activités.*

\section{NOTES}

1. Aux fins de cette recherche, nous adoptons la définition de la performance proposée par Morin, Savoie et Beaudin (1994). Ces auteurs définissent la performance organisationnelle comme un jugement prononcé par les multiples groupes de pression sur les activités, les produits, les résultats ou les effets de l'organisation.

2. Nous entendons, par " conceptions ", de petites théories du sens commun utiles au quotidien, issues de connaissances pratiques et théoriques.

3. Système de couplage souple est la traduction de l'expression loose coupling introduite par Weick en 1976. Cet auteur écrit : " loose coupling is a situation in which elements are responsive, but retain evidence of separateness and identity » (p. 3). Le terme coupling représente la stabilité, la cohérence, l'union, les liens entre les différents éléments et leur degré de détermination. Le terme loosely représente l'autonomie, le changement, l'indépendance des éléments et leur capacité à changer rapidement. L’image qui en résulte est un système qui peut être en même temps : « ouvert et fermé », " rationnel et indéterminé », « spontané et délibéré ».

4. L'évaluation est une « démarche permettant de porter un jugement, à partir de normes ou de critères établis, sur la valeur d'une situation, d'un processus ou d'un élément donné, en vue d'une décision pédagogique ou administrative. » (Legendre, 2005)

5. Un modèle typologique est un modèle fondé sur une typologie c'est-à-dire « un système de description, de comparaison, de classification ou d'explication des éléments d'un ensemble qui permet de ramener d'une façon simplifiée à quelques types fondamentaux une multitude d'objets. » (Legendre, 2005)

6. La signification de la notion de complexité est loin d'être claire. Comme le note Fenske (1980) «Universities have a complexity and multiplicity of goals compared with a profit-oriented organisation. Their various constituencies and interest groups-academic staff, students, administrators, councils, government, the Ministry, the public, funding bodies, professional groups hold divergent, even opposing views on university goals and goals priorities, both within and between the groups. [...] Higher education is indeed many things to many people: a "mosaic of conflicting values. » 
7. Les experts ont été choisis en fonction des critères suivants : avoir occupé une position stratégique dans le domaine de l'enseignement supérieur, de l'évaluation institutionnelle, de l'administration publique ou posséder une expérience exceptionnelle dans ces domaines; ou, être des professeurs-chercheurs reconnus dans le domaine. Une liste de vingt-cinq experts a ainsi été constituée à partir des propositions des experts rencontrés et en consultant les sites Internet des universités. Un ordre de priorité a été établi et les onze premiers experts disponibles ont été rencontrés.

8. Le comité de validation était formé de l'auteure, de son directeur de recherche, de sa co-directrice de recherche et d'un expert externe.

9. Loi sur les établissements d'enseignement de niveau universitaire, L.R.Q, c E-14.1

\section{RÉFÉRENCES}

Amaral, A., Jones, G.A., \& Karseth, B. (2002). Governing higher education. Dordrecht, Pays-Bas : Kluwer Academic Publishers.

Askling, B., \& Kristensen, B. (2000). Vers l'organisation apprenante : incidences pour le gouvernement et la direction des établissements. Gestion de l'enseignement supérieur, 12(2), 17-44.

Bertrand, D., \& Busugutsala, G.G. (1995). L'université québécoise du troisième type : dynamique vers l'an 2010. Sainte-Foy, Québec : Université du Québec. Groupe de recherche sur l'enseignement supérieur.

Birnbaum, R. (1988). How colleges work: the cybernetics of academic organization and leadership. San Francisco, Californie : Jossey-Bass.

Boltanski, L., \& Thévenot, L. (1991). De lajustification : Les économies de la grandeur. Paris, France : Gallimard.

Bonniol, J.-J., \& Vial, M. (1997). Les modèles de l'évaluation. Paris, France : De Boeck Université.

Bouvier, A. (2007). Territoires et management scolaire. Dans \& (dir.), L'Europe de l'éducation : entre management et politique. Lyon, France : Institut national de recherche pédagogique.

Clark, B.R. (1998). Creating entrepreneurial universities: organizational pathways of transformation. Oxford, Angleterre : Pergamon Press.

Cohen, M.D., March, J.G., \& Olsen, J.P. (1972). A garbage can model of organizational choice. Administrative Science Quarterly, 17(1), 1-25.

Conseil supérieur de l'éducation (CSE). (1999). L'évaluation institutionnelle en éducation : une dynamique propice au développement : rapport annuel 1998-1999 sur l'état et les besoins de l'éducation. Sainte-Foy, Québec : Le Conseil.

Crespo, M., \& Dridi, H. (2007). Intensification of university-industry relationships and its impact on academic research. Higher education, 54, 61-84.

Dill, D. (2003). Le paradoxe de la qualité académique: Implications pour les universités et les politiques publiques. Revue des sciences de l'éducation, 29(2), 337-352.

Duke, C. (1992). The learning University: Toward a new paradigm? Buckingham, Angleterre : Society for research into Higher Education et Open University Press. 
Fédération québécoise des professeures et professeurs d'université (FQPPU). (1997). L'Université comme service public. Communication présentée par le Comité exécutif de la FQPPU au Congrès de la FQPPU, Magog-Orford, Québec.

Fenske, R.H. (1980). Setting institutional goals and objectives. Dans P. Jedamus \& M.W. Peterson et al. (dir.), Improving Academic Management: a handbook of planning and institutional research. San Francisco, CA : Jossey-Bass, 177-199.

Fortin, M.-F. (1996). Le processus de la recherche, de la conception à la réalisation. Montréal, Québec : Décarie éditeur.

Freitag, M. (2002). L'université aujourd'hui : Les enjeux du maintien de sa mission institutionnelle d'orientation de la société. Dans Gilles Gagné (dir.), Main basse sur l'éducation. Montréal, Québec : Éditions Nota bene.

Garvin, D. (1993). Building a learning organization. Harvard Business Revue, $71(4), 7884$.

Harvey, L., \& Knight, P. (1996). Transforming higher education. Londres, Angleterre : Open University Press.

Héon, L. (1991). Les forces du marché et de la stratification universitaires sur l'évolution des programmes de premier cycle (le cas des baccalauréats en administration des affaires à l'UQAM et à l'École des H.E.C. (Thèse de doctorat inédite). Université Laval.

James, R. (2000). L'assurance qualité et le difficile problème de la gestion du savoir organisationnel en milieu universitaire. Gestion de l'enseignement supérieur, 12(3), 45-64.

Kerlinger, F.N. (1973). Foundations of Behavioral Research (2 éd.). New York, NY : Holt, Rinehart and Winston, Inc.

Legendre, R. (2005). Dictionnaire actuel de l'éducation. Montréal, Québec : Guérin.

Lemaître, M.J. (2002). Quality as Politics. Quality in Higher Education, 8(1), 29-37.

Lucier, P. (2006). L'université québécoise. Sainte-Foy, Québec : Presses de l'Université Laval.

Mead, P. (1995). Utilising the university as a learning organization to facilitate quality improvement. Quality in Higher education, 1(2), 111-121.

Ministère de l'Éducation du Québec. (2000). Politique québécoise à l'égard des universités : Pour mieux assurer notre avenir collectif. Québec, Québec : Gouvernement du Québec.

Mintzberg, H. (1990). Le management : voyage au centre des organisations. Paris, France : Éditions d'Organisation.

Mintzberg, H., Ahlstrand, B., \& Lampel, J. (1999). Safari en pays stratégie : l'exploration des grands courants de la pensée stratégique. Paris, France : Village mondial. Laval.

Morgan, G. (1999). Images de l’organisation. Québec, Québec : Presses de l’Université

Morin, E. M., Savoie, A., \& Beaudin, G. (1994). L'efficacité de l'organisation : théories, représentations et mesures. Montréal, Québec : Gaëtan Morin. 
Nadeau, G.G., \& Lavigne, J.-C. (1992). Critères et indicateurs de qualité et d'excellence dans les collèges et les universités du Canada. Sainte-Foy, Québec : Université du Québec. Groupe de recherche sur l'enseignement supérieur.

Perrenoud, P. (1995). Des savoirs aux compétences : De quoi parle-t-on en parlant des compétences. Pédagogie collégiale, 9(1), 20-24.

Porter, M.E. (1993). L'avantage concurrentiel des nations. Paris, France : InterÉditions.

Quinn, R.E., \& Rohrbaugh, J. (1983). A spatial model of effectiveness criteria: towards a competing values approach to organizational analysis. Management Science, 29(3), 363 .

Ramsden, P., \& Martin, E. (1996). Recognition of good university teaching: Policies from an Australian study. Studies in Higher Education, 16(2), 129-150.

Rocher, G. (1990). Redéfinition du rôle de l'université. Dans F. Dumont \& Y. Martin (dir.), L'éducation 25 ans plus tard! Et après? (p. 181-198). Québec, Québec : Institut québécois de recherche sur la culture.

Sauvé, L. (1992). Éléments d'une théorie du design pédagogique en éducation relative à l'environnement : Élaboration d'un supramodèle pédagogique. (Thèse de doctorat inédite). Université du Québec à Montréal.

Savard, D., Larouche, C., \& Héon, L. (2004). L'évaluation etla rechercheinstitutionnelles en éducation. Bulletin de la Société québécoise d'évaluation de programme, 17(2), 5.

Silvern, L.C. (1972). System engineering applied to training. Houston, TX : Gulf publishing company.

Srikanthan, G., \& Dalrymple, J.F. (2002). Developing a holistic model for quality in higher education. Quality in Higher Education, 8(3), 215-224.

Tavenas, F. (Ed.). (2004). Assurance qualité : Référentiel partagé d’indicateurs et de procédures d'évaluation. Bruxelles, Belgique : Association Européenne de l'Université.

Weick, K.E. (1976). Educational organizations as loosely coupled systems. Administrative Science Quarterly, 21(1), 1-19.

\section{CONTACT INFORMATION}

Catherine Larouche

Département des sciences de l'éducation

Université du Québec à Chicoutimi

Chicoutimi, Québec G7H 2B1

catherine_larouche@uqac.ca

Catherine Larouche est avocate et professeure en administration scolaire à l'Université du Québec à Chicoutimi. Elle est titulaire d'un Doctorat en Administration et évaluation en éducation de l'Université Laval. Elle a reçu le prix George-L-Geis pour sa thèse intitulée : La validation d'une typologie des universités en vue d'évaluer leur performance. 
Denis Savard est professeur en évaluation et recherche institutionnelles au département des Fondements et pratiques en éducation de l’Université Laval.

Lucie Héon est professeure en planification et gestion de l'éducation au département des Fondements et pratiques en éducation de l'Université Laval.

Jean-Joseph Moisset est professeur émérite de l’Université Laval. 